\title{
Route to enhance the efficiency of organic photovoltaic solar cells - by adding ferroelectric nanoparticles to P3HT/PCBM admixture
}

\author{
David Black, Iulia Salaoru, and Shashi Paul ${ }^{\mathrm{a}}$ \\ Emerging Technologies Research Centre, Hawthorn Building, De Montfort University, The Gateway, \\ Leicester LE1 9BH, UK
}

Received: 14 May 2014 / Received in final form 12 October 2014 / Accepted: 15 October 2014

Published online: 2 December 2014

(C) Black et al., published by EDP Sciences, 2014

\begin{abstract}
We have demonstrated that by adding ferroelectric nanoparticles to poly(3-hexylthiophene) (P3HT) and [6,6]-phenyl- $\mathrm{C}_{61}$-butyric acid methyl ester (PCBM) photovoltaic devices the relative efficiency can be increased compared to the same blend without these nanoparticles. In this work samples of $20 \mathrm{mg} / \mathrm{ml}$ concentrations of $\mathrm{P} 3 \mathrm{HT}$ and PCBM were prepared in a 1:1 ratio and the samples prepared using ferroelectric barium titanate (BT) and strontium titanate (ST) nanoparticles in a 1:1:0.5 ratio. The samples were spin coated onto ITO coated glass with a layer of poly(3,4-ethylenedioxythiophene)poly(styrenesulfonate) (PEDOT:PSS). A top electrode of aluminium $1 \mathrm{~cm}^{2}$ was deposited. The current-voltage characteristics of the devices were determined using a solar simulator and the absorption characteristics by UV-Vis spectroscopy. The samples with BT and ST exhibited increased absorption around $490 \mathrm{~nm}$ and increased open circuit voltage and short circuit current compared to the control P3HT/PCBM sample. The possible mechanism that helps to understand the increase in open circuit voltage and short circuit current is also proposed in this work.
\end{abstract}

\section{Introduction}

The most efficient bulk heterojunction photovoltaic devices comprise blends of poly(3-hexylthiophene) (P3HT) and $[6,6]$-phenyl- $\mathrm{C}_{61}$-butyric acid methyl ester (PCBM) and the best P3HT/PCBM heterojunction devices currently have power conversion efficiencies of around $6 \%$ and quantum efficiencies approaching 100\% [1]. In this work we have made an attempt, for the first time, to demonstrate a relative improvement in the efficiency of the devices by adding ferroelectric nanoparticles to the P3HT/PCBM blends. We have previously demonstrated that by adding ferroelectric nanoparticles, such as barium titanate into polymers, can have a significant effect on dielectric constant of the insulating polymers [2] and the photoconductivity of organic photoconductor materials $[3,4]$. We are anticipating, but not yet fully demonstrated, that the increase in permittivity is also present in the photoconductive polymers doped with ferroelectric nanoparticles.

As it has already been established by a number of workers that organic photovoltaic (OPV) devices are excitonic in nature, which is to say that in conventional

\footnotetext{
${ }^{a}$ e-mail: spaul@dmu.ac.uk
}

semiconductor (CSC) cell the incident photon creates free carriers immediately once absorbed whereas the excitonic semiconductor (XSC) creates electrostatically bound charge carriers called excitons. Whether a material behaves conventionally or excitonically is strongly dependent upon the electrical permittivity of the material. It is possible to determine whether a material will behave as an $\mathrm{XSC}$ or a CSC by comparing the ratios of the Bohr radius with the Coulomb radius. Therefore let the ratio of $r_{c}$ to $r_{B}$ be called $\gamma$.

$$
\gamma=\frac{r_{c}}{r_{B}} \approx\left(\frac{q^{2}}{4 \pi \varepsilon_{0} k_{B} r_{0} m_{e}}\right)\left(\frac{m_{e f f}}{\varepsilon^{2} T}\right) .
$$

A value of $\gamma>1$ indicates that the material will exhibit XSC behaviour and conversely a value of $\gamma<1$ indicates a material that will behave as a CSC.

Where $r_{0}=0.53 \AA$ and is the distance between the electron and the nucleus for hydrogen like structures such as silicon and excitons, $m_{e}$ is the mass of a free electron in a vacuum and $m_{\text {eff }}$ is the effective mass of the electron in the semiconductor, $q$ is the charge on the electron, $\varepsilon$ is the relative permittivity of the material and $r_{c}$ is the critical distance between the charges, $k_{B}$ is Boltzmann's constant and $T$ is the temperature of the system. The material will 
behave in an excitonic manner if $r_{c}>r_{B}$ and where $r_{B}$ is greater than the radius of the particle [5]. It is important to mention here two important caveats to this model; firstly $m_{\text {eff }}$ is not well defined in XSC materials due to the fact that charge transport generally occurs due to a hopping mechanism rather than delocalised band transport, secondly $\varepsilon$ is a bulk property of the material and tends towards the permittivity of free space at small scales [5].

Persson and Iganas [6] have suggested a seven step process of charge generation in XSC materials:

1. Photon incoupling.

2. Photon absorption.

3. Exciton formation.

4. Exciton migration.

5. Exciton dissociation.

6. Charge transport.

7. Charge collection.

Although it can be argued that processes 1 and 2 are essentially the same and this could be a six step process this description is essentially accurate and corresponds with the work of Moliton and Nunzi [7]. Once an exciton has formed (step 3 ) it is free to move within the polymer matrix, however the distance that an exciton can travel is limited to a few tens of nanometers before they recombine. It is essential therefore that they can dissociate before they recombine. The object of this research is to attempt to maximize the dissociative region of the material and hence increase the overall electron harvest by maximizing the amount of excitons that dissociate into charge carriers.

In bulk heterojunction devices charge separation occurs when an exciton travels to an interface between the photoconductive polymer where the exciton is produced and the electron accepting nanoparticles in the polymer matrix, in this case P3HT and PCBM, respectively. At the interface between the two materials an electrochemical potential is set up that is equal to the difference between the Fermi levels for each material [8]. This potential results in an electron flow from the PCBM to the polymer, which results in a depleted region surrounded by positively charged polymer, due to an excess of holes and a negatively charged PCBM molecules due to an excess of electrons. Once an equilibrium state is reached an electric field is set up which is essential for charge separation [8]. Exciton dissociation occurs if the Coulomb forces binding the exciton are less than the built in field of the junction $\left(q \Phi_{b i}\right)$.

Therefore by increasing the relative permittivity of the bulk material we can influence at least two important factors in exciton dissociation and hence charge production, namely; increasing permittivity decreases the Coulomb potential in the exciton, and increases the Debye length of the material, which is related to the width of the depletion region [6], and hence to the dissociative region [8].

In this work we have added ferroelectric barium titanate (BT) and strontium titanate (ST) to blends of PCBM and P3HT. The commercially available BT and ST nanoparticles are cubic in nature and not ferroelectric in this state. By annealing these nanoparticles in
Table 1. Polymer-nanoparticle blends used in active layers.

\begin{tabular}{ccccc}
\hline Sample & $\begin{array}{c}\text { P3HT } \\
(\mathrm{mg} / \mathrm{ml})\end{array}$ & $\begin{array}{c}\text { PCBM } \\
(\mathrm{mg} / \mathrm{ml})\end{array}$ & $\begin{array}{c}\mathrm{BT} \\
(\mathrm{mg} / \mathrm{ml})\end{array}$ & $\begin{array}{c}\mathrm{ST} \\
(\mathrm{mg} / \mathrm{ml})\end{array}$ \\
\hline 1 & 20 & 20 & 0 & 0 \\
2 & 30 & 30 & 0 & 0 \\
3 & 40 & 40 & 0 & 0 \\
4 & 20 & 20 & 0 & 10 \\
5 & 20 & 20 & 10 & 0 \\
\hline
\end{tabular}

air at $1000{ }^{\circ} \mathrm{C}$ the structure of the molecules was altered from cubic to tetragonal and hence to a ferroelectric state prior to inclusion in the blend [9-11]. The samples were electrically tested in our solar simulator and their absorption characteristics were measured using UV-Vis spectroscopy. The fill factor (FF) and power conversion efficiency (PCE) were calculated from the current-voltage $(I-V)[12]$ characterisitics.

\section{Experiment}

A blend of P3HT with varying concentration ferroelectric nanoparticles was prepared to understand the effect on dielectric constant. The dielectric constant was deduced from capacitance-voltage measurement of metalblend-metal structures.

Three sets of sample solar cells were fabricated with different concentrations of P3HT, PCBM and while keeping strontium titanate (average size $<60 \mathrm{~nm}$ ) and barium titanate nanoparticles (average size $<70 \mathrm{~nm}$ ) concentrations $(10 \mathrm{mg} / \mathrm{ml})$ the same. The base solution for each concentration was a blend of P3HT and PCBM, to which was added either no nanoparticles to form a control sample, ST or BT. The concentrations for the $\mathrm{P} 3 \mathrm{HT} / \mathrm{PCBM}$ samples were; 20,30 and $40 \mathrm{mg} / \mathrm{ml}$ in a $1: 1$ ratio. TheP3HT/PCBM/nanoparticle blend was a 1:1:0.5 ratio. The concentrations of the samples are detailed in Table 1. Samples 1, 2 and 3 were compared initially to determine the ideal concentration, and then the samples containing the ferroelectric nanoparticles were prepared and compared with the control sample for that concentration.

The schematic of various structures of the samples is shown in Figure 1.

The devices were prepared by first spin coating a layer of poly(3,4-ethylenedioxythiophene)poly(styrenesulfonate) (PEDOT:PSS) $(\sim 30 \mathrm{~nm})$ onto cleaned ITO $(\sim 80 \mathrm{~nm})$ coated glass substrate. This followed by the deposition of P3HT/PCBM active layer $(\sim 100 \mathrm{~nm})$ by spin coating. Aluminium top electrodes, $1 \mathrm{~cm}^{2}$ area, were deposited by thermal evaporation at a chamber pressure of $\sim 1 \times 10^{-6}$ mbar.

Electrical tests were carried out on the samples using standard current-voltage $(I-V)$ tests in both illuminated and non-illuminated conditions. The illumination used was an Oriel AM1.5 light source, equivalent to $\sim 8.58 \mathrm{~mW} / \mathrm{cm}^{2}$. 
UV-Vis measurements were carried out on samples deposited on ITO coated glass substrates using the Thermo Scientific Evolution 300 spectrometer.

\section{Results and discussion}

It has been known for some time that by adding high permittivity (high $k$ ) materials, particularly ceramics, to polymers and epoxy resins that the permittivity of the blend of materials will be higher than that of the original polymer or epoxy alone [13-18]. A number of models have been proposed which describe the effective permittivity $\left(\varepsilon_{\text {eff }}\right)$ of the composite material and a few of which are presented and compared below. The simplest model is known as the volume fraction average model and is a summation of the relative fractions of each type of material by volume. This method is generally held to be inaccurate, but can be used for a quick first approximation [14].

$$
\varepsilon_{\text {eff }}=\phi_{1} \varepsilon_{1}+\phi_{2} \varepsilon_{2}
$$

where; $\phi$ is the volume fraction, $\varepsilon$ is the permittivity and the subscripts 1 and 2 represent the polymer and ceramic material, respectively. This model predicts a sharp increase in permittivity at a relatively small fraction of ceramic filler, which in practice does not occur [14]. More accurate models are based on "mean field theory" which reduces all interactions on a body to a single average interaction thus removing the uncertainty generally associated with a many body problem. The Maxwell-Garnett equation is one such model $[14,18]$ which can be written as:

$$
\varepsilon_{e f f}=\varepsilon_{1} \frac{\varepsilon_{2}+2 \varepsilon_{1}-2\left(1-\phi_{1}\right)\left(\varepsilon_{1}-\varepsilon_{2}\right)}{\varepsilon_{2}+2 \varepsilon_{1}+\left(1-\phi_{1}\right)\left(\varepsilon_{1}-\varepsilon_{2}\right)} .
$$

This is valid for a single ceramic or ferroelectric particle surrounded by polymer. This equation also has a drawback in that as it is valid only when the filler fraction is infinitesimally small.

The Bruggeman model is another mean field theory model that treats the polymer/ceramic matrix as a series of repeated units of spherical ceramics surrounded by polymer. This can be written as $[14,18]$ :

$$
\phi_{1}\left(\frac{\varepsilon_{1}-\varepsilon_{\text {eff }}}{\varepsilon_{1}+2 \varepsilon_{\text {eff }}}\right)+\phi_{2}\left(\frac{\varepsilon_{2}-\varepsilon_{\text {eff }}}{\varepsilon_{2}+2 \varepsilon_{\text {eff }}}\right)=0 .
$$

In this model the value of the dielectric constant increases dramatically for ceramic filler volumes of $20 \%$ and above. The two final equations compared here are the logarithmic Lichtnecker equation:

$$
\ln \varepsilon_{e f f}=\phi_{2} \ln \varepsilon_{2}+\left(1-\phi_{2}\right) \ln \varepsilon_{1}
$$

and the "effective medium theory (EMT) model" that describes the permittivity of a two part system in terms of the relative fractions of the components:

$$
\varepsilon_{\text {eff }}=\varepsilon_{m}\left[1+\frac{\phi_{2}\left(\varepsilon_{2}-\varepsilon_{1}\right)}{\varepsilon_{1}+\left(n\left(1-\phi_{2}\right)\left(\varepsilon_{2}-\varepsilon_{1}\right)\right)}\right]
$$

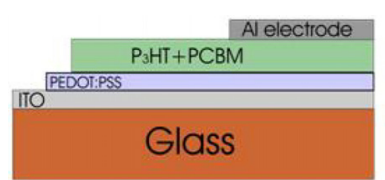

(a)

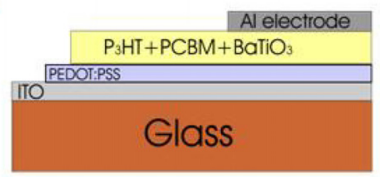

(C)

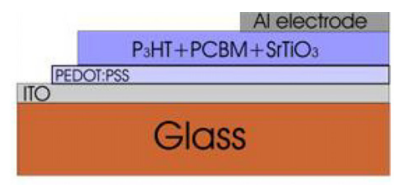

(b)
Fig. 1. Typical structure of organic heterojunction solar cells as used in this work, (a) control sample 1 of $\mathrm{P} 3 \mathrm{HT} / \mathrm{PCBM}$, (b) sample 2, P3HT/PCBM plus strontium titanate and (c) $\mathrm{P} 3 \mathrm{HT} / \mathrm{PCBM}$ plus barium titanate.

where $n$ is a correction factor which compensates for the non spherical nature of the ceramics and is generally less than 0.15 for a polymer/ceramic matrix.

To obtain comparative permittivity measurements for samples of P3HT containing varying quantities of barium and strontium titanate. The first set of samples used the capacitance measurements and after some optimisation produced reliable results. It is thought that the problems in making reliable measurements with this technique were twofold, firstly any minor imperfection in the layer such as pinholes could cause the device to fail and secondly the any clumping of nanoparticles could provide a path for electrical conduction. Also high leakage currents can lead to unusual results such as negative capacitances and unusually high or low results [19-21]. Once uniform films were produced after optimisation of various parameters, the majority of these issues disappeared and reliable measurements could be taken. In order to overcome the difficulties in making these measurements the polymer/nanoparticle solution was left in the ultrasonic bath for a minimum of 8 hours to maximise the dispersion of the nanoparticles in the polymer.

Given that barium titanate and strontium titanate caused an increase in photoconductivity both were investigated to determine what, if any, effect they would have on the permittivity of P3HT.

Figure 2 clearly demonstrates an almost linear increase in the permittivity of $\mathrm{P} 3 \mathrm{HT}$ with increasing volume fraction of barium titanate. The upper limit of this effect has not been investigated as the concentration levels required to determine this would be far in excess of the levels required to fabricate functional photovoltaic devices. The EMT model fits the data well with an $R^{2}$ value of 0.925 . The measured value of permittivity for the closest result to $20 \mathrm{mg} / \mathrm{ml}$ concentration is 8.24 compared to 7.74 as predicted by the model.

Figure 3 shows the results of the permittivity measurements for samples of P3HT with varying concentrations of strontium titanate. The increase in permittivity in this case is slightly higher than that produced by barium titanate with similar levels of concentration, which could be expected from the fact that strontium titanate is a 


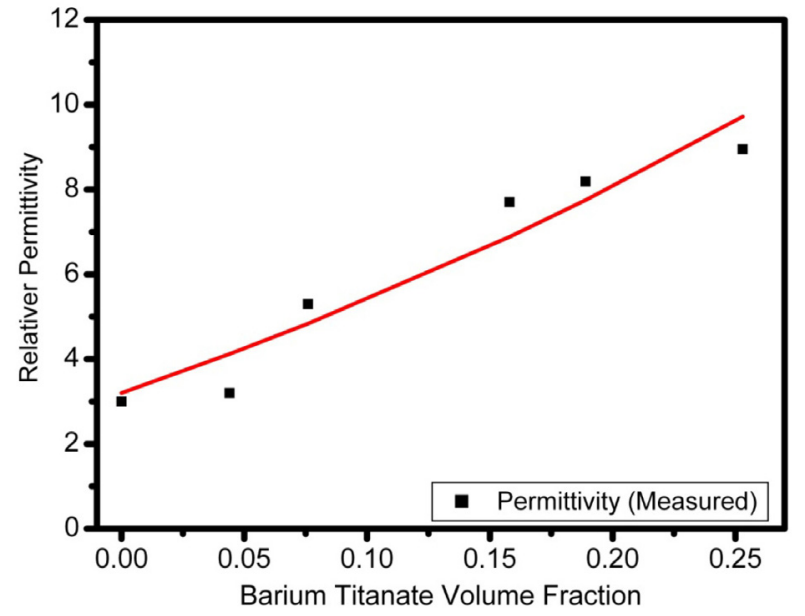

Fig. 2. Relative permittivity of P3HT with increasing volume fractions of barium titanate.

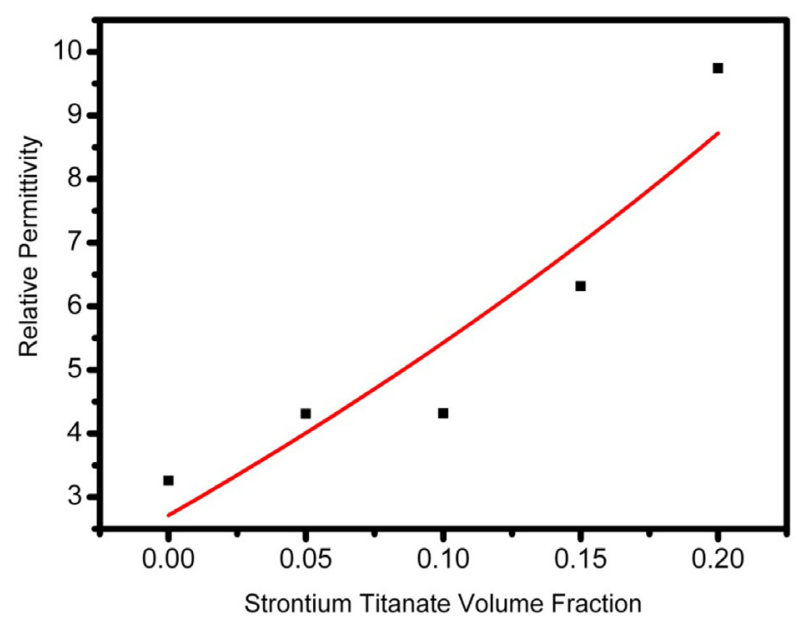

Fig. 3. Relative permittivity of P3HT with increasing volume fractions of Strontium Titanate.

higher $k$ value to begin with. These data confirm that both barium and strontium titanates are suitable candidate materials to be added to $\mathrm{P} 3 \mathrm{HT} / \mathrm{PCBM}$ blends to potentially increase the efficiency of this type of solar cell.

The first test of photoconductivity using P3HT films used only barium titanate, although subsequent tests also included strontium titanate. Figure 4 shows the results of the first test, with a base $20 \mathrm{mg} / \mathrm{ml}$ solution of P3HT was divided into two vials, one was kept as a control and the other was added to pre-measured barium titanate, which produced a solution with a concentration of BT of $20 \mathrm{mg} / \mathrm{ml}$. The solution was ultrasonicated until the BT was in full suspension and then spin coated onto glass. Top metal electrodes were deposited to measure the photoconductivity.

The sample containing BT (blue line), clearly improves this to $\sim 1.1 \times 10^{-7} \mathrm{~A}$ and also reduces the hysteresis in the curve, producing an almost linear state. It is clearly evident from the data gather for dielectric constant of P3HT as a function of ferroelectric nanoparticles concentration and its effect on the photoconductivity may re-

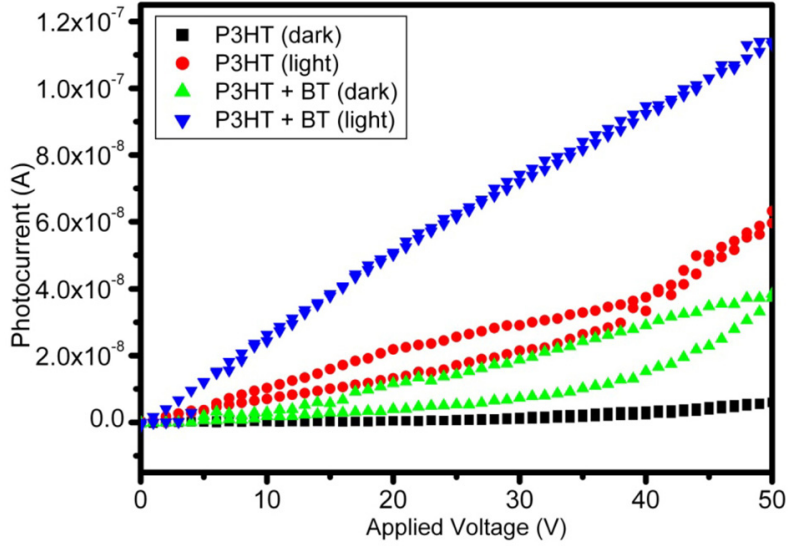

Fig. 4. Photoconductivity measurements for P3HT samples containing $20 \mathrm{mg} / \mathrm{ml}$ barium titanate.

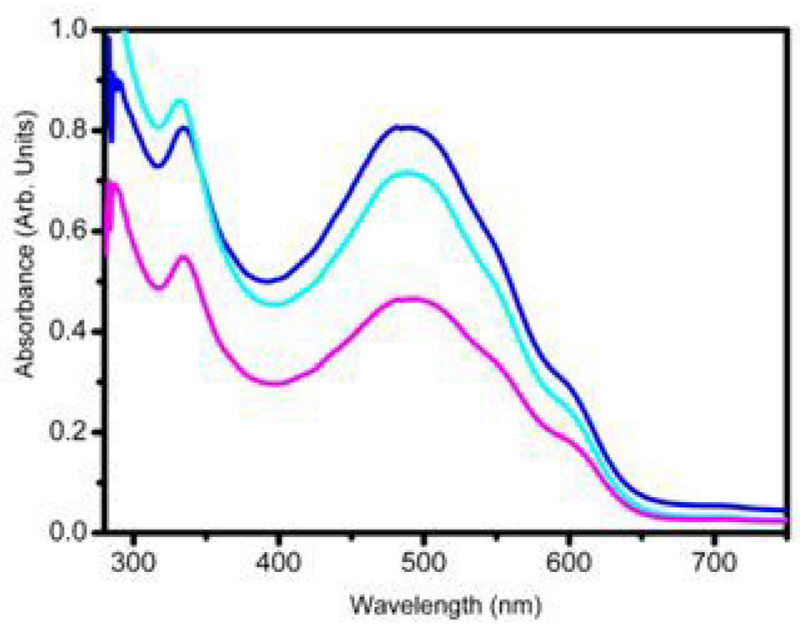

Fig. 5. UV-Vis spectrum of sample 1 (magenta), sample 4 (cyan) and sample 5 (blue) for comparison.

sult in enhanced efficiency of organic photovoltaic solar cells. The clear role of ferroelectric nano-particles need further investigation, for example, measurement of charge carrier life time, etc. of thin film containing ferroelectric nano-particles.

The UV-Vis spectroscopy measurements shown in Figure 5 suggest that samples 1,4 and 5 have absorption peaks at two different energy values; the first around $334 \mathrm{~nm}$ and the second at $490 \mathrm{~nm}$. The first peak is slightly shifted to $331 \mathrm{~nm}$ in sample 4 containing strontium titanate but remains at $334 \mathrm{~nm}$ in the other two samples. The $490 \mathrm{~nm}$ peaks correspond in all three samples and agree with other works with regard to the location of the peaks in the green part of the spectrum. The absorption shoulders that are expected between 500 and $600 \mathrm{~nm}$ are not very pronounced in these samples. This may be a characteristic of the solvent which in this case was chloroform. Samples with chlorobenzene based solvents have been shown by Vanlaeke et al. to show flatter regions in this area [22]. It can clearly be seen that the relative absorbance of both polymer blends containing ferroelectric 
D. Black et al.: Route to enhance the efficiency of organic photovoltaic solar cells

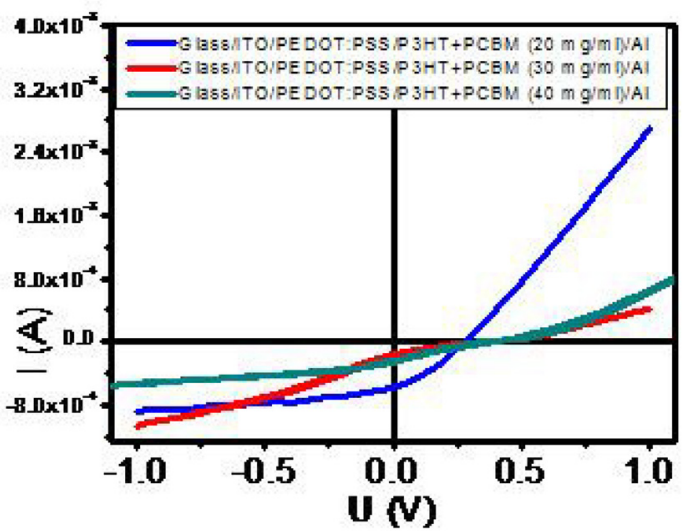

Fig. 6. Comparison of $I-V$ characteristics of P3HT/PCBM samples with 20,30 and $40 \mathrm{mg} / \mathrm{ml}$ concentrations in a 1:1 ratio.

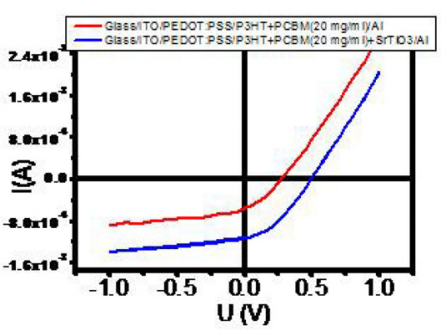

(a)

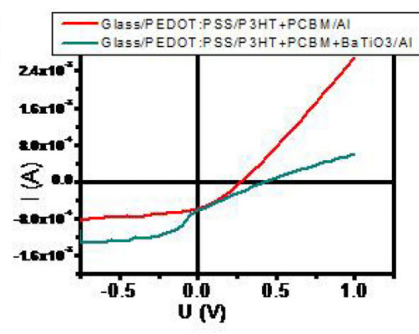

(b)
Fig. 7. $I-V$ characteristics of (a) sample 4 (blue) compared with sample 1 (red) and (b) sample 5 (green) compared with sample 1 (red).

nanoparticles is greater than those of $\mathrm{P} 3 \mathrm{HT} / \mathrm{PCBM}$ alone. We believe that this link to the increase in the dielectric constant of the P3HT/PCBM system by addition of ferroelectric nano-particles, which might increase the Debye length and longer availability of the charge carriers in admixture and further investigation is needed to confirm this.

The comparison of samples 1, 2 and 3 is presented in Figure 6. It is clear from both the shape of the curves and the fill factor and efficiency that sample $1(20 \mathrm{mg} / \mathrm{ml})$ has the highest overall efficiency. The open circuit voltage is higher for both samples 2 and 3 , but the fill factor is reduced for these samples as there is an increased effect from resistances that is causing flattening of the curves. Form these initial results the decision was taken to use a base solution of $20 \mathrm{mg} / \mathrm{ml}$ and to add the ferroelectric nanoparticles to this solution. The solution used was from the same batch as solution 1 and the nanoparticles were added as described above.

The $I-V$ characteristics of samples 1,4 and 5 are presented and compared in Figure 7. Figure 7a shows a comparison between samples 1 and 4 . Both samples exhibit curves of similar shape, presenting the expected diode like behaviour. Sample 4, containing ST clearly produces higher open circuit voltage and short circuit current than sample 1. From the theory stated above one explanation for the increase in current is the increased numbers of
Table 2. Results of electrical measurements for $1 \mathrm{~cm}^{2}$ devices.

\begin{tabular}{ccccc}
\hline Sample & $V_{\mathrm{OC}}(\mathrm{V})$ & $I_{\mathrm{SC}}(\mathrm{mA})$ & $\mathrm{FF}$ & $\mathrm{PCE} \%$ \\
\hline 1 & 0.27 & 0.50 & 0.38889 & 0.63 \\
2 & 0.33 & 0.18 & 0.16111 & 0.11 \\
3 & 0.40 & 0.27 & 0.15167 & 0.20 \\
4 & 0.48 & 1.10 & 0.37652 & 2.37 \\
5 & 0.43 & 0.70 & 0.19565 & 0.70 \\
\hline
\end{tabular}

excitons that can dissociate into charge carriers, due to both a decrease in Coulomb forces and an increase in the volume of the dissociation region. The reason for the increase in open circuit voltage is less clear but is likely to be linked to the ferroelectric particles increasing the built in potential of the devices $[23,24]$. We anticipate additional measurements in the near future to confirm the increase in permittivity of the material and to determine the variations in the built in potential.

Figure $7 \mathrm{~b}$ shows the comparison between samples 1 and 5 , as with sample 4 the open circuit voltage and short circuit current are increased, but the curve of sample 5 does not retain the expected ideal diode like shape compared to sample 1 . In any solar cell, XSC or CSC, there are two resistances; a series resistance $\left(r_{s}\right)$ related to the contact and bulk resistances and a shunt resistance $\left(r_{s h}\right)$ arising from the contact alone, in an ideal device $r_{s}=0$ and $r_{s h}=\infty$. As $r_{s}$ increases and $r_{s h}$ decreases the diode moves away from perfect diode like behaviour and the shape of the curve is flattened. For reasons not currently clear the resistances in the BT sample are obviously affecting the behaviour of the material, why this is not evident in the ST sample is also not known at present.

The $V_{\mathrm{oc}}, I_{\mathrm{sc}}$, fill factor and PCE of different samples are tabulated in Table 2.

While the overall efficiencies of these devices are not high compared to the best devices currently produced by other research groups, the relative efficiencies are compared in this work. Sample 4 presents an overall PCE of $2.37 \%$ which is almost a factor of four greater than the control sample. Even though the resistances have affected the diode characteristics of sample 5 it still has a higher PCE than the control. Strontium titanate, shows great promise as an additive to improve the performance of OPV devices as does barium titanate if the resistances in the devices can be overcome. It is hoped that by continuing to use ferroelectric nanoparticles in these devices that we can improve their efficiency compared to P3HT/PCBM devices alone.

\section{Conclusions}

We have demonstrated that out of three concentrations of P3HT/PCBM the most efficient and closest to an ideal diode is $20 \mathrm{mg} / \mathrm{ml}$. We have also shown that it is possible to improve the efficiency of P3HT/PCBM OPV devices by adding ferroelectric nanoparticles such as strontium 
titanate or barium titanate to the active layer. The maximum efficiency obtained was by the sample containing strontium titanate, which was four times more efficient than the P3HT/PCBM control sample. The sample with barium titanate was more efficient than the control, but was limited by the fact that the resistances in the cell reduced the ideal diode like characteristics of this device. Ferroelectric nanoparticles may offer one route towards the efficient organic photovoltaic devices.

Authors would like to thank EPSRC for DTA student funding.

\section{References}

1. S.H. Park, A. Roy, S. Beaupre, S. Cho, N. Coates, J.-S. Moon, D. Moses, M. Leclerc, K. Lee, A.J. Heeger, Nat. Photon. 3, 297 (2009)

2. D. Black, S. Paul, I. Salaoru, J. Nanosci. Nanotechnol. Lett. 2, 1 (2010)

3. D. Black, S. Paul, Mater. Res. Soc. Symposium Proc. 1303, 69 (2011)

4. S. Paul, D. Black, Organic photoconductive material (Patent application number GB2484743 - Filing date: 23 October 2010, Publication 25 April 2012)

5. B.A. Gregg, Coulomb Forces in Excitonic Solar Cells, in Organic Photovoltaics: Mechanisms, Materials and Devices, edited by S.S. Sun, N.S. Sariciftci (Taylor and Francis, Boca Raton, 2005), pp. 139-159

6. N.-K. Persson, O. Inganas, Simulations of Optical Processes in Organic Photovoltaic Devices, in Organic Photovoltaics: Mechanisms, Materials and Devices, edited by S.S. Sun, N.S. Sariciftci (Taylor and Francis, Boca Raton, 2005), pp. 107-138
7. A. Moliton, J.-M. Nunzi, Polymer Int. 55, 583 (2006)

8. E. Kymakis, G.A.J. Amaratunga, Solar Cells Based on Composites of Donor Conjugated Polymers and Carbon Nanotubes, in Organic Photovoltaics: Mechanisms, Materials and Devices, edited by S.-S. Sun, N.S. Sariciftci (Taylor and Francis, Boca Raton, 2005)

9. L.M.B. Alldredge et al., Appl. Phys. Lett. 94, 052904 (2009)

10. T.-I. Yang, P. Kofinas, Polymer 48, 791 (2007)

11. I. Salaoru, S. Paul, Philos. Trans. Roy. Soc. A 367, 4227 (2009)

12. J. Nelson, The Physics of Solar Cells, 1st edn. (Imperial College Press, 2003)

13. P. Kim et al., Adv. Mater. 19, 1001 (2007)

14. P. Barber et al., Materials 2, 1697 (2009)

15. Z.-M. Dang et al., Mater. Chem. Phys. 109, 1 (2008)

16. S. George, M.T. Sebastian, Comput. Sci. Technol. 69, 1298 (2009)

17. S. Ogitani, S.A. Bidstrup-Allen, P.A. Kohl, Adv. Packag. IEEE Trans. 23, 313 (2000)

18. G. Subodh et al., Appl. Phys. Lett. 95, 062903 (2009)

19. G. Arlt, D. Hennings, G. de With, J. Appl. Phys. 58, 1619 (1985)

20. A.K. Jonscher, in Dielectric Relaxation in Solids (Chelsea Dielectrics Press, London, 1996), p. 380

21. B. Pradhan, A.J. Pal, Chem. Phys. Lett. 416, 327 (2005)

22. P. Vanlaeke, G. Vanhoyland, T. Aernouts, D. Cheyns, C. Deibel, J. Manca, P. Heremans, J. Poortmans, Thin Solid Films 511-512, 358 (2006)

23. C.J. Brabec, A. Cravino, D. Meissner, N.S. Sariciftci, T. Fromherz, M.T. Rispens, L. Sanchez, J.C. Hummelen, Adv. Funct. Mater. 11, 374 (2001)

24. M.F. Lo, T.W. Ng, T.Z. Liu, V.A.L. Roy, S.L. Lai, M.K. Fung, C.S. Lee, S.T. Lee, Appl. Phys. Lett. 96, 113303 (2010)

Cite this article as: David Black, Iulia Salaoru, Shashi Paul, Route to enhance the efficiency of organic photovoltaic solar cells - by adding ferroelectric nanoparticles to P3HT/PCBM admixture, EPJ Photovoltaics 5, 50403 (2014). 